\title{
COPD depicted - patients drawing their lungs
}

This article was published in the following Dove Press journal:

International Journal of COPD

3 November 2017

Number of times this article has been viewed

\author{
Ad A Kaptein' \\ Jitske Tiemensma ${ }^{2}$ \\ Elizabeth Broadbent ${ }^{3}$ \\ Guus M Asijee ${ }^{4,5}$ \\ Maarten Voorhaar ${ }^{4,5}$ \\ 'Medical Psychology, Leiden University \\ Medical Centre (LUMC), Leiden, \\ the Netherlands; ${ }^{2}$ Psychological \\ Sciences, University of California, \\ Merced, CA, USA; ${ }^{3}$ Psychology \\ Department, Auckland University \\ Medical School, Auckland, New \\ Zealand; ${ }^{4}$ CAPHRI School for \\ Public Health and Primary Care, \\ Maastricht University, Maastricht, \\ the Netherlands; ${ }^{5}$ Boehringer \\ Ingelheim Pharma GmbH \& Co. KG, \\ Ingelheim am Rhein, Germany
}

Correspondence: Ad A Kaptein Medical Psychology, Leiden University Medical Centre LUMC, PO Box 9600 2300 RB Leiden, the Netherlands Email a.a.kaptein@lumc.nl
Background: Given the increasing importance of patient-reported outcomes (PRO) in quality medical care, we examined the value and feasibility of an innovative method for assessing patients' illness perceptions, represented in drawings made by patients with COPD of their lungs.

Aim: The aim of our study was: to study patients' representation of COPD as reflected in their drawings of their lungs; and to examine scores on a validated measure that assesses illness perceptions (ie, Brief Illness Perception Questionnaire [B-IPQ]).

Patients and methods: One hundred outpatients with COPD, mean age 70 years, selected from a pharmacy database, participated and 98 filled out the B-IPQ. Eighty-seven patients completed the drawing task.

Results: The illness perceptions as reflected in the responses to the B-IPQ scales represented a quite optimistic view of COPD and its consequences. The drawings of the lungs reflected a considerable discordance between patients' representations and medically accepted representations of lungs of a person with COPD.

Conclusion: Assessing illness perceptions in clinical care and research about COPD offers opportunities to identify goals for patient education and self-management. Inviting patients to draw their illness is an innovative and promising approach to assessing PRO.

Keywords: COPD, drawings, illness perceptions, PRO, quality of life, self-management

\section{Introduction}

Assessing the nature and severity of COPD is fundamental to delivering quality medical care for patients with COPD. ${ }^{1}$ However, the objective severity of the disease is only partly associated with its impact on patients' daily life. ${ }^{2}$ Rather, patients' perceptions of their disease (ie, their illness perceptions) drive how they cope with the illness, their quality of life (QoL), and their behavioral responses to the illness and its consequences (ie, self-management). ${ }^{3}$ It follows that addressing maladaptive, unhelpful illness perceptions in clinical care for patients with COPD translates into more adequate coping, self-management skills, and QoL. ${ }^{4,5}$ In patients with other chronic illnesses, evidence supports the value of changing unhelpful illness perceptions to more adaptive and constructive perceptions and achieving positive outcomes (eg, for patients with myocardial infarction; irritable bowel syndrome; hemodialysis; cardiovascular diseases). ${ }^{7-10}$ We reviewed the topic of self-management and the importance of illness perceptions in persons with COPD in a previous paper in this journal. ${ }^{6}$

Given the important role of illness perceptions in persons with a chronic condition, assessing illness perceptions in a valid and reliable manner is essential. Various methods of assessing illness perceptions are available, with questionnaires as the major approach (see www.uib.no/ipq for extensive information on questionnaires assessing illness perceptions). ${ }^{11,12}$ An innovative method of assessing illness perceptions is asking patients to make a drawing of their illness. The earliest studies 
on patients drawing their illness date back some 50 years (eg, where patients with psychiatric diagnoses drew their representations of depression and other psychiatric conditions). ${ }^{13}$ More recently, drawings have been utilized in virtually all categories of chronic somatic diseases (eg, ranging from asthma to vaginal thrush), in children (with cystic fibrosis [CF], for instance) and adults (eg, with lung cancer). ${ }^{14,5}$ In the area of chronic respiratory diseases, only a handful of drawing studies are available. Three studies depict asthma: two in children, one in adults. ${ }^{17-19}$ All three studies clearly show that asthmatic episodes have a great emotional impact. A moving drawing by a child with $\mathrm{CF}$ depicts dying due to $\mathrm{CF} .{ }^{14}$ Hoogerwerf et al asked patients with lung cancer "to make a drawing of what they imagined their diseased lungs look like", therefore, comparing "subjective" with "objective" representations of a disease. ${ }^{15}$ The patients' drawings more or less reflected the image of their chest X-rays. Luthy et al asked patients with COPD "to draw their body, and to represent limitations and difficulties related to breathing". ${ }^{16}$ The drawings revealed a major gap between patients' views and "objective reality", depicted in textbooks on lung diseases.

The aim of this study was to examine patients' perceptions of their COPD as reflected in their drawings, and to examine scores on a validated questionnaire assessing illness perceptions (ie, Brief Illness Perception Questionnaire $[\mathrm{B}-\mathrm{IPQ}])^{20}$

\section{Methods}

\section{Patients}

Patients were recruited in pharmacies in the Netherlands. ${ }^{3}$ Patients were invited to the pharmacy and handed an information leaflet and an informed consent form. After signing the written informed consent form, patients were handed a booklet with questionnaires and their medication data were extracted from the pharmacist's database.

The study was approved by the Medical Ethics Committee of the Leiden University Medical Centre (protocol number P11.170).

One hundred patients, 58 males, 42 females, all $>40$ years of age (mean age $70[ \pm 10]$ years), filled out the booklet. Seventy-five percent of the patients reported having received a diagnosis of COPD more than 3 years ago. On a scale from 0 (not severe) to 100 (very severe), the patients indicated a mean perceived severity of their COPD of $39( \pm 26)$. Patients reported using on average two types of medication, with 97\% using tiotropium, $42 \%$ salmeterol, $20 \%$ fluticasone, and $19 \%$ formoterol (see Tiemensma et al for further details). ${ }^{3}$
On a scale from 0 (poor) to 100 (good), patients rated their quality of life as $70( \pm 25)$. A third of the patients had a "low" level of education (primary school), another third "medium" (high school), and another third a "high" level of education (at least college degree).

Patients were included if they used COPD maintenance medication therapy for 1 year or longer, were aged 40 years or older, and had been diagnosed with COPD at least 1 year ago. Patients were cared for in a general practice/family medicine setting, where pulmonary function tests are not carried out. Global Initiative for Chronic Obstructive Lung Disease (GOLD) categories are not available although it is safe to assume that the patients were GOLD I, II or III.

\section{Measures}

Sociodemographic, clinical, and psychological data were collected via the booklet. Illness perceptions were assessed used the B-IPQ, which has eight single-item scales and an open-ended question about the perceived causes of the illness. ${ }^{20}$ The B-IPQ was designed to rapidly assess cognitive and emotional representations of an illness. The first eight items are scored on a $0-10$ scale. The B-IPQ has been used in diverse age groups, illness types, and languages. A recent meta-analysis has shown that the scale has good concurrent validity and predictive validity. ${ }^{21}$ In addition, patients were asked to:

Draw how you imagine your lungs to be. We are not interested in your drawing talent, a simple sketch is fine. We are interested in your ideas about what your lungs look like.

The drawings were examined by the researchers for the emergence of themes.

\section{Results}

The items of the B-IPQ are shown in Table 1, with the mean score per item.

A table of illness perception scores from a number of other clinical populations in the recent systematic review by Broadbent et al allows comparison of the scores from this sample with those with acute conditions (eg, cold) and other chronic illnesses (eg, cancer, rheumatoid arthritis, diabetes). ${ }^{21}$ The mean scores on the B-IPQ items in Table 1 seem to reflect a somewhat optimistic view of the consequences of the illness and the options to control it.

Of the 87 patients who created a drawing, anatomical drawings were made by 61 patients. Text was added in 12 drawings (eg, "inflammation and junk" in the lungs); four reflected 
Table I B-IPQ scores

\begin{tabular}{|c|c|}
\hline B-IPQ item & $\begin{array}{l}\text { Average score } \\
(\mathrm{n}=100 \text { patients } \\
\text { with COPD) } \\
\end{array}$ \\
\hline $\begin{array}{l}\text { How much does your illness affect your life? } \\
\text { ("0" no effects at all - severely affects my life "I0") }\end{array}$ & 5.03 \\
\hline $\begin{array}{l}\text { How long do you think your illness will continue? } \\
\text { ("0" a very short time - forever "10") }\end{array}$ & 9.42 \\
\hline $\begin{array}{l}\text { How much control do you feel you have over } \\
\text { your illness? } \\
\text { ("0" absolutely no control - extreme amount } \\
\text { of control "10") }\end{array}$ & 6.17 \\
\hline $\begin{array}{l}\text { How much do you think your treatment } \\
\text { can help your illness? } \\
\text { ("0" not at all - extremely helpful "I0") }\end{array}$ & 6.95 \\
\hline $\begin{array}{l}\text { How much do you experience symptoms } \\
\text { from your illness? } \\
\text { ("0" no symptoms at all - many severe } \\
\text { symptoms "I0") }\end{array}$ & 5.52 \\
\hline $\begin{array}{l}\text { How concerned are you about your illness? } \\
\text { ("0" not at all concerned - extremely } \\
\text { concerned "10") }\end{array}$ & 5.03 \\
\hline $\begin{array}{l}\text { How well do you feel you understand your illness? } \\
\text { ("0" don't understand at all - understand very } \\
\text { clearly "I0") }\end{array}$ & 7.54 \\
\hline $\begin{array}{l}\text { How much does your illness affect you emotionally } \\
\text { (eg, does it make you angry, scared, upset, } \\
\text { or depressed?) } \\
\text { ("0" not at all affected emotionally - extremely } \\
\text { affected emotionally "I0") }\end{array}$ & 4.43 \\
\hline
\end{tabular}

Abbreviation: B-IPQ, Brief Illness Perception Questionnaire.

emotion (drawing 21 in Figure 1, with the cigarettes in the lungs); ten patients wrote a question mark or "no idea"; 13 patients did not draw anything at all and left the box provided completely empty.

Examples of the drawings are given in Figure 1. In the drawings, various degrees of detail, emotion, and anatomical correctness are visible. The drawings made by patients 26 and 30 (Figure 1A) indicate good anatomical correctness. The drawings made by patients 37 and 14 (Figure 1B) indicate relatively little knowledge. The drawings made by patients 21 and 56 (Figure 1C) show symbolic representations, with the drawing made by patient 21 the only drawing where cigarettes are depicted and an understanding of cigarettes as a causal factor. The drawings made by patients 34 and 38 (Figure 1D) show unstructured images.

The number of drawings in our study was relatively small and the degree of detail in drawings produced was insufficient to examine associations between drawing characteristics and other variables (eg, age, sex, severity of COPD, B-IPQ scores). Larger samples are therefore indicated. The drawings produced by the patients in our study are quite unstructured.
As emphasized in the instructions in the booklet, the researchers were not interested in drawing skills. Nevertheless, only a small percentage of patients in the sample drew lungs with at least some degree of anatomical correctness. This contrasts with their relatively good self-reported understanding of their illness $(7.5 / 10)$.

\section{Discussion}

Asking patients to draw their illness is cheaper and quicker than performing a pulmonary function assessment; it produces patient-reported outcome data. Drawings can quite likely bypass issues of social desirability. Both adults and young children can draw their representations of their medical problems, thereby giving the physician access to their personal views (for a systematic review of drawing research see Broadbent et al). ${ }^{22}$ Drawings can also be used as a way to assess patients' perceptions of changes as a consequence of medical treatment (eg, a study on changes in drawings of the heart before and after a cardiac rehabilitation program). ${ }^{23}$ Furthermore, drawings have been used to diagnose patient problems that did not come to light via other more traditional methods. ${ }^{24,25}$ Many patients with chronic conditions such as COPD are rather limited in their health literacy; they often fail to fully understand questions or the results of medical assessments. For these patients, drawing their illness is a relatively straightforward, practical method to express perceptions of their health, which may otherwise be left unspoken. ${ }^{26}$

This is the first study to ask patients with COPD to draw their perceptions of their lungs. The Luthy et al study instructed the respondents with COPD to draw their body, not explicitly their lungs. ${ }^{16}$ The Hoogerwerf et al study asked patients with lung cancer to draw their diseased lungs. ${ }^{15}$ Given the prevalence of COPD, it is rather surprising to find that this disorder is not more often represented in drawing research. The recent systematic review on drawing research by Broadbent et al lists some 100 studies with patients drawing their illness. ${ }^{22}$ Two drawing studies on lungs (or $2 \%$ of studies in the review) contrast rather dramatically with the $10 \%$ prevalence of COPD in populations around the world. This discrepancy seems to be rather consistent with the overall underrepresentation of behavioral research about persons with COPD, compared to, for instance, persons with cardiovascular diseases or cancer. ${ }^{6}$

Having COPD implies breathlessness as a major symptom, in addition to a range of other symptoms (eg, depression, fatigue, social isolation). ${ }^{5,6}$ Breathlessness is not a topic that is explicitly represented in the drawings of the patients in the current study. It is quite likely that the drawing instruction 
A

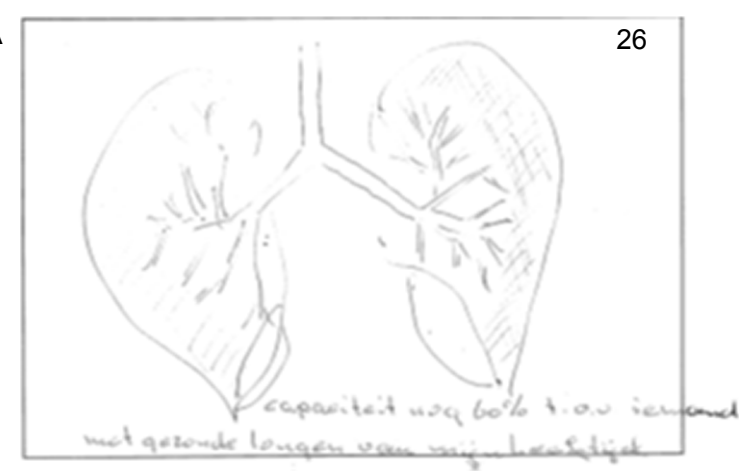

B

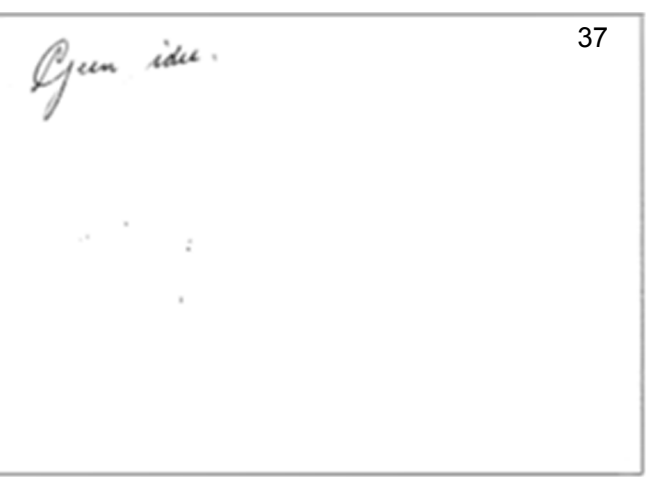

C

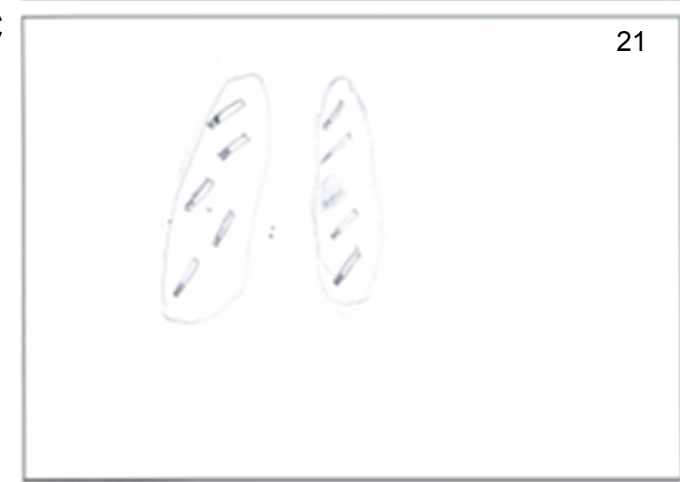

D

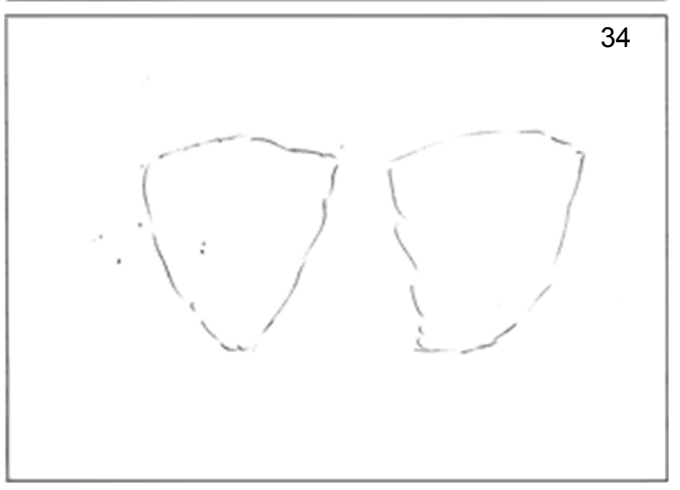

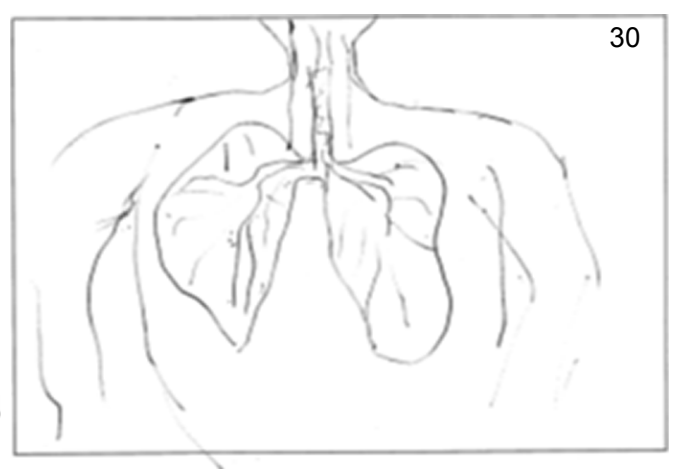
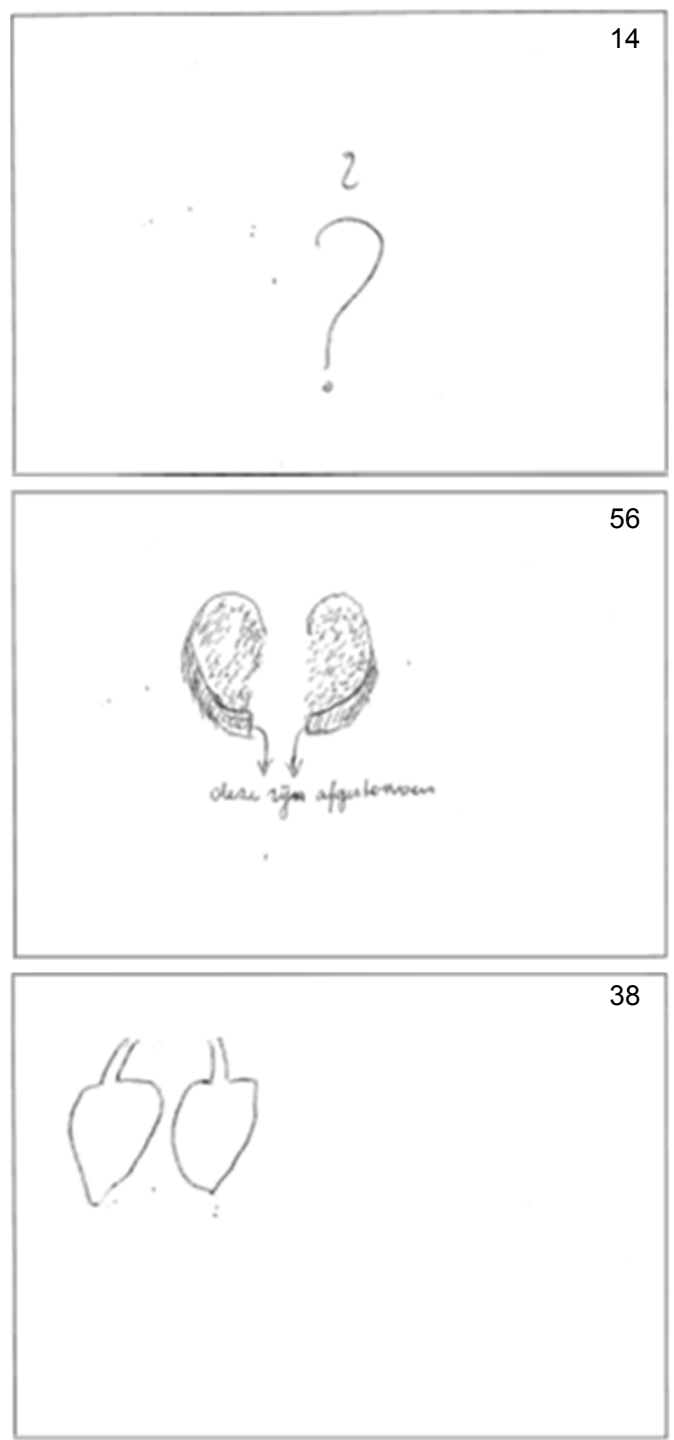

Figure I Examples of patients' drawings.

Notes: (A) Anatomically correct; (B) absence of knowledge; (C) symbolic representation; (D) unstructured representation. Text in 26 : "capacity $60 \%$ compared to someone of my age with healthy lungs"; in 37: "no idea"; in 56: "these have died".

("draw how you imagine your lungs to be [...] we are interested in your ideas about what your lungs look like") encouraged patients to try to draw a factual, anatomical representation of lungs, rather than a symbolic representation of symptoms that patients associate with their COPD. In other studies, patients' drawings explicitly show symbols of strong emotions, for instance of being scared because of attacks of asthma (ropes encircling the thorax) ${ }^{19}$ daggers and thunder bolts in migraine, ${ }^{28}$ and blood and symbols of death in myocardial infarction. ${ }^{23}$ Future studies should consider using instructions to also draw emotions associated with having COPD. 
In drawing research, empirical studies on associations between clinical characteristics of an illness, and drawings and illness perceptions are lacking. It is, therefore, hard to draw conclusions about whether the range of disease severity in our study is associated with drawing characteristics or scores on the B-IPQ. This is another area of future research. Clinical observation and empirical work point to the importance of denial, stigma, embarrassment, and shame regarding a long career of smoking tobacco as a major cause of COPD. In our study, the patient who drew cigarette butts in his lungs is one of the few who apparently acknowledged smoking as being related to the disease.

Behavioral scientists from "the Denver group" were one of the first to point out the relevance of stigma in persons with COPD ${ }^{29}$ Recently, Rose et al reviewed the topic of stigma-related experiences in non-communicable respiratory disease and underlined associations between stigma on the one hand, and reductions in quality of life in the patients concerned, on the other. ${ }^{30}$

The limitation of the relatively small number of drawings does not take away strength in this study: the majority of patients were willing and able (although not all) to draw their lungs and their respiratory illness. Future research in this area may benefit from expanding and refining clinical (eg, COPD severity) and psychological characteristics (eg, depression, self-efficacy) that might be associated with aspects of the drawing. ${ }^{3}$ In intervention studies - be they with a pharmacological, clinical or behavioral focus - drawings could be included as an additional outcome measure (compare with Reynolds et al and Broadbent et al where drawings were predictive of important outcomes such as return to work). ${ }^{7,23}$ Drawings are an additional way of studying patients' perceptions of COPD, which may be of additional value to tests of pulmonary function, laboratory data, or chest X-ray, namely reflecting how patients perceive and cope with the condition. Furthermore, using drawings instead of using questionnaires may close the gap for those patients who have limited health literacy. ${ }^{26}$ Future research might examine the effects of focused attention by health care providers to educate patients about how COPD affects the lungs and other physical issues. ${ }^{27}$ Greater patient understanding of how COPD anatomically affects the lungs and understanding the influence of smoking and medication on lung function may affect patients' adherence to medication or smoking cessation. ${ }^{31}$

\section{Conclusion}

Asking patients to draw their illness and report their illness perceptions may highlight areas of misunderstanding or lack of knowledge to clinicians. Clinicians could then target these areas for greater discussion and education using anatomical drawings, computer animations, or plastic models of the lungs. This may increase patients' feelings of selfefficacy and improve self-management, resulting in better quality of life.

\section{Disclosure}

GMA and MV are employees of Boehringer Ingelheim Pharma GmbH \& Co. KG, Ingelheim am Rhein, Germany. AAK, JT and EB report no conflicts of interest in this work.

\section{References}

1. goldcopd.org [homepage on the Internet]. GOLD - From the Global Strategy for the Diagnosis, Management and Prevention of COPD, Global Initiative for Chronic Obstructive Lung Disease (GOLD); 2017. Available from: http://goldcopd.org/. Accessed September 25, 2017.

2. Liu Y, Li H, Ding N, Wang N, Wen D. Functional status assessment of patients with COPD: a systematic review of performance-based measures and patient-reported measures. Medicine (Baltimore). 2016; 95(20):e3672.

3. Tiemensma J, Gaab E, Voorhaar M, Asijee G, Kaptein AA. Illness perceptions and coping determine quality of life in COPD patients. Int J Chron Obstruct Pulmon Dis. 2016;11:2001-2007.

4. Atkins CJ, Kaplan RM, Timms RM, Reinsch S, Lofback K. Behavioral exercise programs in the management of chronic obstructive pulmonary disease. J Consult Clin Psychol. 1984;52(4):591-603.

5. Kaptein AA, Scharloo M, Fischer MJ, et al. Illness perceptions and COPD: An emerging field for COPD patient management. J Asthma. 2008;45(8):625-629.

6. Kaptein AA, Fischer MJ, Scharloo M. Self-management in patients with COPD: theoretical context, content, outcomes, and integration into clinical care. Int J Chron Obstruct Pulmon Dis. 2014;9:907-917.

7. Broadbent E, Ellis CJ, Thomas J, Gamble G, Petrie KJ. Further development of an illness perception intervention for myocardial infarction patients: a randomized controlled trial. J Psychosom Res. 2009;67(1): 17-23.

8. Chilcot J, Moss-Morris R. Changes in illness-related cognitions rather than distress mediate improvements in irritable bowel syndrome (IBS) symptoms and disability following a brief cognitive behavioural therapy intervention. Behav Res Ther. 2013;51(10):690-695.

9. Jansen DL, Heijmans M, Rijken M, Kaptein AA. The development of and first experiences with a behavioural self-management intervention for end-stage renal disease patients and their partners. J Health Psychol. 2011;16(2):274-283.

10. Petrie KJ, Cameron LD, Ellis CJ, Buick D, Weinman J. Changing illness perceptions after myocardial infarction: an early intervention randomized controlled trial. Psychosom Med. 2002;64(4): 580-586.

11. Kaptein AA, Broadbent E. Illness cognition assessment. In: Ayers S, Baum A, McManus C, et al, editors. Cambridge Handbook of Psychology, Health \& Medicine. 2nd ed. Cambridge: Cambridge University Press; 2007:268-273.

12. Kaptein AA, Scharloo M, Weinman JA. Assessment of illness perceptions. In: Vingerhoets A, editor. Assessment in behavioral medicine. Hove, UK: Brunner-Routledge; 2001:179-194.

13. Wadeson H, Carpenter WT Jr. A comparative study of art expression of schizophrenic, unipolar depressive, and bipolar manic-depressive patients. J Nerv Mental Dis. 1976;162(5):334-344.

14. Tsanakas JN. Perception of death. Arch Dis Child. 2004;89(12):1083.

15. Hoogerwerf MA, Ninaber MK, Willems LNA, Kaptein AA. "Feelings are facts": illness perceptions in patients with lung cancer. Respir Med. 2012;106(8):1170-1176. 
16. Luthy C, Cedraschi C, Pasquina P, Uldry C, Perron NJ, Janssens JP. Perception of chronic respiratory impairment in patients' drawings. J Rehabil Med. 2013;45(7):694-700.

17. Gabriels RL, Wamboldt MZ, McCormick DR, Adams TL, McTaggart SR. Children's illness drawings and asthma symptom awareness. J Asthma. 2000;37(7):565-574.

18. Quinn CM. Children's asthma: new approaches, new understandings. Ann Allergy. 1988;60(4):283-292.

19. Cheung MM, Saini B, Smith L. Drawing asthma: an exploration of patients' perceptions and experiences. J Asthma. Epub 2017 May 17.

20. Broadbent E, Petrie KJ, Main J, Weinman J. The brief illness perception questionnaire. J Psychosom Res. 2006;60(6):631-637.

21. Broadbent E, Wilkes C, Koschwanez H, Weinman J, Norton S, Petrie KJ. A systematic review and meta-analysis of the Brief Illness Perception Questionnaire. Psychol Health. 2015;30(11):1361-1385.

22. Broadbent E, Schoones JW, Tiemensma J, Kaptein AA. Drawings in illness - systematic review. Health Psychol Rev. In press 2017.

23. Reynolds L, Broadbent E, Ellis CJ, Gamble G, Petrie KJ. Patients' drawings illustrate psychological and functional status in heart failure. J Psychosom Res. 2007;63(5):525-532.

24. Di Gallo A, Kuehne T. Ghost in the tree. Lancet. 2008;372(9649): 1570 .

25. Tiemensma J, Pereira AM, Romijn JA, Broadbent E, Biermasz NR, Kaptein AA. Persistent negative illness perceptions despite long-term biochemical control of acromegaly: novel application of the drawing test. Eur J Endocrinol. 2015;172(5):583-593.
26. Puente-Maestu L, Calle M, Rodriguez-Hermosa JL, et al. Health literacy and health outcomes in chronic obstructive pulmonary disease. Respir Med. 2016;115:78-82.

27. Weldam SW, Lammers JW, Heijmans MJ, Schuurmans MJ. Perceived quality of life in chronic obstructive pulmonary disease patients: a crosssectional study in primary care on the role of illness perceptions. $B M C$ Fam Pract. 2014;15:140.

28. Rutberg S, Ohrling K. Migraine - more than a headache: women's experiences of living with migraine. Disabil Rehab. 2012;34(4): 329-336.

29. Kinsman RA, Yaroush RA, Fernandez E, Dirks JF, Schocket M, Fukuhara J. Symptoms and experiences in chronic bronchitis and emphysema. Chest. 1983;83(5):755-761.

30. Rose S, Paul C, Boyes A, Kelly B, Roach D. Stigma-related experiences in non-communicable respiratory diseases: a systematic review. Chron Respir Dis. 2017;14(3):199-216.

31. Stephens MH, Grey A, Fernandez J, Kalluru R, Faasse K, Horne A, Petrie KJ. 3-D bone models to improve treatment initiation among patients with osteoporosis: A randomized controlled pilot trial. Psychol Health. 2016;31(4):487-497.
International Journal of COPD

\section{Publish your work in this journal}

The International Journal of COPD is an international, peer-reviewed journal of therapeutics and pharmacology focusing on concise rapid reporting of clinical studies and reviews in COPD. Special focus is given to the pathophysiological processes underlying the disease, intervention programs, patient focused education, and self management protocols.

\section{Dovepress}

This journal is indexed on PubMed Central, MedLine and CAS. The manuscript management system is completely online and includes a very quick and fair peer-review system, which is all easy to use. Visit $\mathrm{http}: / / \mathrm{www}$.dovepress.com/testimonials.php to read real quotes from published authors. 